\title{
Carrier frequency and symbol rate estimation based on cyclic spectrum
}

\author{
CAO Sisi and ZHANG Weiyan* \\ Institute of Computer Applications, China Academy of Engineering Physics, Mianyang 621000, China
}

\begin{abstract}
Carrier frequency and symbol rate estimation are the main contents of parameter estimation, which is the basis of modulation recognition and further processing of signals especially in non-cooperative communication. With the development of wireless communication, the signal transmission environment has become increasingly bad, causing more difficulties in parameter estimation. It is well known that the signal cycle spectrum is robust to noises and signal parameters are closely related. In practice, it is impossible to calculate the cyclic spectrum of infinite length data signals. When using finite length data to obtain a cycle spectrum, the truncation noise is induced, resulting in interference. It is necessary to overcome the influence of noises in order to improve the detection ability of discrete spectral lines. An improved method of the discrete spectral line extraction algorithm is proposed by reflecting the amplitude advantage of discrete spectral lines through salient features of continuous noises in discrete spectral line neighborhood.
\end{abstract}

Keywords: digital modulation signal, carrier frequency, symbol rate, cyclic spectrum, noise control.

DOI: $10.21629 / J S E E .2020 .01 .05$

\section{Introduction}

Automatic modulation identification and parameter estimation of digital communication signals are the key technologies in communication systems. This process is between signal detection and demodulation, and is of great significance in the fields of cooperative and noncooperative communication such as radio spectrum management and electronic warfare [1-8]. In communication systems, with the effect of modulation and transmission channels, the received signal ineluctably has some distortion. If the receiver wants to monitor, intercept and further process the signals to obtain communication content under unknown conditions, it is necessary to demodulate the signals. The parameters estimation including carrier

\footnotetext{
Manuscript received January 21, 2019.

*Corresponding author.

This work was supported by the National Key R\&D Program of China (2016YFB0800203).
}

frequency and symbol rate is the basis of modulation recognition and demodulation. In recent years, with the rapid development of modern communication and signal processing technologies, more sophisticated signal detection parameter estimation/modulation identification algorithms have been proposed to detect the existence of the target signal as well as obtaining important characteristics of the target signal [9-15].

Studies have been made by researchers on accurate parameter estimation. At present, carrier frequency estimation algorithms can be divided into the following three categories, time-domain, frequency-domain and spectraldomain. The first one is time-domain, such as the zerocrossing method. This method is greatly affected by noises, so the practical application is limited [16]. Secondly, algorithms based on the discrete Fourier transform (DFT) are widely used to estimate carrier frequency. In [17], the idea of the frequency domain estimation algorithm is searching the peak of the spectral peak position, and a novel estimation method based on self-correlation for quadrature phase shift keying (QPSK) signal was proposed. At first, self-correlation biquadratic QPSK signal was analyzed, and then its carrier frequency was estimated through the modified rife (M-rife) algorithm instead of estimating frequency only by the max frequency line position. The simulation results indicate that our carrier frequency estimation method is feasible and more accurate than the former method, and the root of mean square error (RMSE) of the modified method is close to Cramer-Rao lower bound (CRLB). Zaplata et al. [18] introduced a novel approach to the frequency estimation suited for weak suppressed carrier binary phase shift keying (BPSK) signals even below the noise level. The variance of the spectrum averaging frequency estimator and the maximum likelihood (ML) estimator are presented. It is found that the variance of the spectrum averaging frequency estimator is worse compared to the ML estimator above about $-3 \mathrm{~dB}$ of bit energy to noise level ratio. However, in very noisy environment 
the proposed algorithm keeps the variance lower in [18]. Thirdly, it is well known that signal cycle spectrum is robust to noises and the signal parameters which are closely related [19]. In [20], a novel algorithm was proposed to improve the accuracy in frequency estimation by transforming the character of cyclic autocorrelation correlation to the frequency domain to highlight the effective signal spectral, and tune the effective carrier frequency spectrum. In [21], a novel method of estimating carrier frequency and chip rate in severe signal environments was presented, based on the characteristics of cyclostationarity of multiple phase shift keying (MPSK) signals and the spectral lines on the bifrequency surface. This method, with a high estimating accuracy, has been proved by computer simulations.

About the symbol rate estimation, Liu et al. [22] proposed two approaches to the estimate symbol rate for multi-channel digital modulation signals mixed linearly, namely wavelet transform (WT) and twice WT. In [22], WT and twice WT were utilized in multi-channel linearly mixed signals after frequency conversion, respectively, to detect the jump border of mixed signals. Then we take Fourier transform to coefficients of WT. Symbol rate information can be extracted from spectrum of WT coefficients. Computer simulations are performed for common digital modulation signals. Communication signal cyclic spectrum density cyclic frequency domain contains a carrier frequency, symbol rate and other modulation parameter information, for stationary noise and interference have a good inhibitory effect [23-25].

The research is motivated by previous studies [24,25] in which we propose a new approach based on the cyclic spectrum and modified discrete spectral line extraction to estimate the modulation parameters. The paper is organized as follows. Section 2 gives the signal model. Section 3 explains the signal parameter estimation by the cyclic spectrum. Section 4 presents the simulation results and analysis. Finally, Section 5 is the conclusion.

\section{Signal model and theory of cyclic spectrum}

\subsection{Signal model}

The received signal can be defined as

$$
x(t)=s(t)+n(t)
$$

where $s(t)$ can be amplitude modulation (AM), MPSK or orthogonal frequency division multiplexing (OFDM) signals, and $n(t)$ is the additive zero-mean Gaussian noise. $s(t)$ can be expressed as

$$
s(t)=A_{k} g\left(t-k T_{s}\right) \exp \left[-\mathrm{j} 2 \pi f_{c} t+\phi(t)\right]
$$

where $A_{k}$ is the signal amplitude; $g(t)$ is the pulse shape function; $T_{s}$ is the symbol period; $f_{c}$ is the unknown carrier frequency; $\phi(t)$ is the signal phase.
After modulation, shaping filter and sampling, statistical characteristics of digital signals typically have a certain periodicity, called cyclostationarity [26].

\subsection{Theory of cyclic spectrum}

It is well known that most of communication signals are cyclostationary, and cyclostationary features can be used in signal detection [27], parameter estimation and modulation detection. The cyclic spectrum theory is depicted simply below.

$x(t)$ is a complex sinusoidal signal with the mean zero and random noise $n(t)$. The cyclic autocorrelation of a complex-valued time series $x(t)$ is defined by

$$
R_{x}(t, \tau)=\mathrm{E}\left\{x(t+\tau / 2) x^{*}(t-\tau / 2)\right\}
$$

where $R_{x}(t, \tau)$ is the autocorrelation function of $x(t)$. If $x(t+\tau / 2) x^{*}(t-\tau / 2)$ has statistical properties, and the period is $T_{0}$. Then

$$
\begin{gathered}
R_{x}(t, \tau)=\lim _{N \rightarrow \infty} \frac{1}{2 N+1} . \\
\sum_{n=-N}^{N} x\left(t+n T_{0}+\tau / 2\right) x^{*}\left(t+n T_{0}-\tau / 2\right)
\end{gathered}
$$

where $1 / T_{0}$ is the symbol rate. Do its Fourier series expansion as

$$
R_{x}(t, \tau)=\sum_{m=-\infty}^{\infty} R_{x}^{\alpha}(\tau) \mathrm{e}^{\mathrm{j} \frac{2 \pi}{T_{0}} m t}=\sum_{m=-\infty}^{\infty} R_{x}^{\alpha}(\tau) \mathrm{e}^{\mathrm{j} 2 \pi \alpha t}
$$

where $\alpha=m / T_{0}$. After Fourier transform,

$$
S_{x}^{\alpha}(f)=\int_{-\infty}^{\infty} R_{x}^{\alpha}(\tau) \mathrm{e}^{-\mathrm{j} 2 \pi f \tau} \mathrm{d} \tau
$$

where $S_{x}^{\alpha}(f)$ is called the cyclic spectrum density function, $\alpha$ is the cyclic frequency and $f$ is the fourier frequency.

\section{Parameter estimation based on cyclic spectrum}

Since stationary noise does not have cyclostationarity in the case where cyclic frequency is not equal to zero, and the theoretical spectrum correlation function is zero [28]. The use of cyclic spectrum correlation in signal analysis and processing can eliminate some random factors coming from the signal itself, such as additive white Gaussian noise. Therefore, in terms of parameter estimation and extraction it shows better anti-interference and high resolution. The cyclic spectral magnitude can be obtained approximately at $\alpha$ section in $f=0$ and $f=f_{c}$ for all signals [29]. The following description is given as an example of MPSK. 


\subsection{Correspondence between parameter and cyclic spectrum}

The MPSK modulation signal model can be expressed as

$$
\begin{aligned}
& x(t)=\operatorname{Re}\left\{x_{1}(t) \mathrm{e}^{\mathrm{j}\left(2 \pi f_{c} t+\varphi_{0}\right)}\right\} \\
& x_{1}(t)=\sum_{n=-\infty}^{\infty} q(t-n T) \mathrm{e}^{\mathrm{j} \theta_{n}}
\end{aligned}
$$

where $x_{1}(t)$ is the complex envelope of $x(t), f_{c}$ is the carrier frequency, $\varphi_{0}$ is the initial phase of the signal, $T$ is the duration of a symbol, and $q(t)$ is the pulse shaping filter. $\theta_{n} \in\{2 \pi(m-1) / M\}(m=1,2, \ldots, M)$.

The general forms of the MPSK signal cyclic spectrum are shown in (9), (10), Fig. 1 and Fig. 2.

When $M=2$,

$$
S_{x}^{\alpha}(f)=\left\{\begin{array}{l}
\frac{1}{4 T}\left[Q\left(f-f_{c}+\frac{\alpha}{2}\right) \cdot Q^{*}\left(f-f_{c}-\frac{\alpha}{2}\right)+Q\left(f+f_{c}+\frac{\alpha}{2}\right) \cdot Q^{*}\left(f+f_{c}-\frac{\alpha}{2}\right)\right], \quad \alpha=\frac{n}{T} \\
\frac{1}{4 T}\left[\mathrm{e}^{\mathrm{j} 2 \phi_{0}} Q\left(f-f_{c}+\frac{\alpha}{2}\right) \cdot Q^{*}\left(f+f_{c}-\frac{\alpha}{2}\right)+\mathrm{e}^{-\mathrm{j} 2 \phi_{0}} Q\left(f+f_{c}+\frac{\alpha}{2}\right) \cdot Q^{*}\left(f-f_{c}-\frac{\alpha}{2}\right)\right], \\
\alpha= \pm 2 f_{c}+\frac{n}{T}
\end{array}\right.
$$

When $M \geqslant 4$,

$$
\begin{aligned}
& S_{x}^{\alpha}(f)=\frac{1}{4 T}\left[Q\left(f-f_{c}+\frac{\alpha}{2}\right) \cdot Q^{*}\left(f-f_{c}-\frac{\alpha}{2}\right)+\right. \\
& \left.Q\left(f+f_{c}+\frac{\alpha}{2}\right) \cdot Q^{*}\left(f+f_{c}-\frac{\alpha}{2}\right)\right], \quad \alpha=\frac{n}{T} .
\end{aligned}
$$

Wherein, $Q(f)$ is the Fourier transform of the shaping pulse $q(t)$. Analyzing the cyclic spectrum magnitude through (9) and (10) for all MPSK signals can acquire two important expressions.

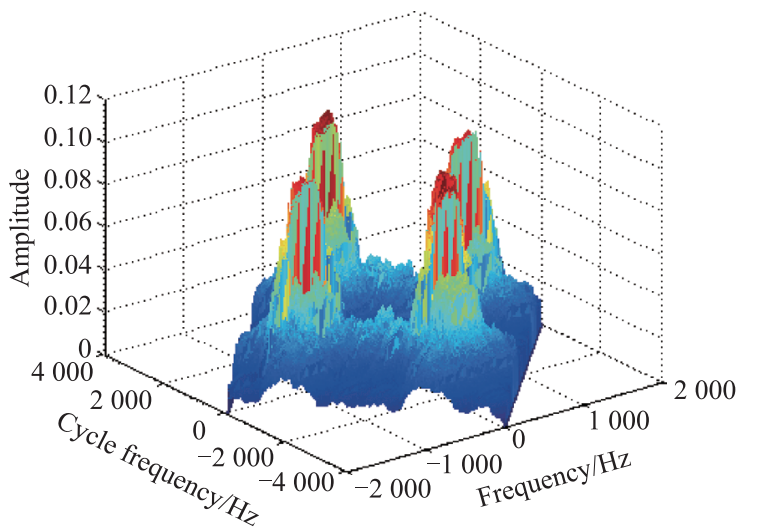

Fig. 1 Cyclic spectrum of BPSK signals

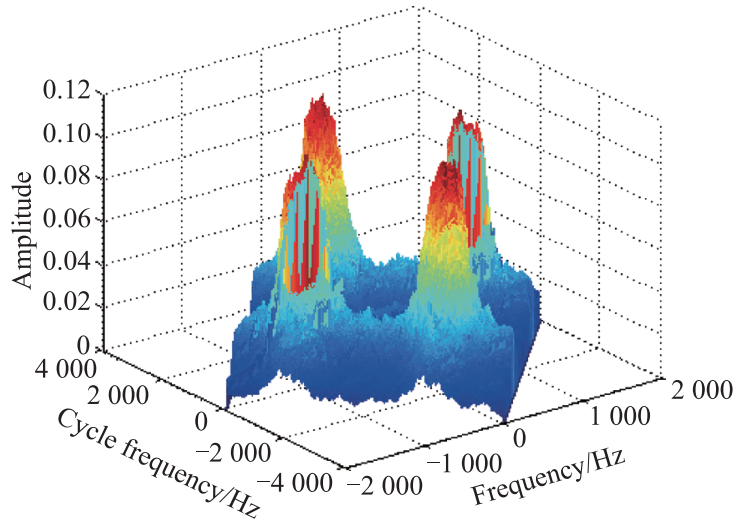

Fig. 2 Cyclic spectrum of QPSK signals $f$-section of the cyclic spectrum when $\alpha=0$ is

$$
\left|S_{x}^{\alpha}(f)\right|_{\alpha=0}=\frac{1}{4 T}\left[\left|Q\left(f-f_{c}\right)\right|^{2}+\left|Q\left(f+f_{c}\right)\right|^{2}\right] .
$$

Due to the characteristic of $Q(f)$, there is a peak value at $f= \pm f_{c}$.

$\alpha$-section of the cyclic spectrum when $f=0$ is

$$
\begin{gathered}
\left|S_{x}^{\alpha}(f=0)\right|= \\
\left\{\begin{array}{l}
\frac{1}{2 T}\left|Q\left(f_{c}-\frac{\alpha}{2}\right) Q^{*}\left(f_{c}+\frac{\alpha}{2}\right)\right|, \quad \alpha=\frac{k}{T} \\
\frac{1}{4 T}\left|Q\left(f_{c} \mp \frac{\alpha}{2}\right)\right|^{2}, \quad \alpha= \pm 2 f_{c}+\frac{k}{T} \\
0, \quad \text { otherwise }
\end{array} .\right.
\end{gathered}
$$

The first item is to be close to a small value of 0 . In the second item, the cyclic spectrum has a peak value when $\alpha= \pm 2 f_{c}$, and the second largest value when $\alpha= \pm 2 f_{c}+1 / T$. For the cyclostationarity of white Gaussian noise, the noise cyclic spectrum is not included here, so we search the maximum value when $f=0$, as shown in Fig. 3. At this point the search to a maximum value corresponding to the cycle frequency, in theory, equals twice the carrier frequency.

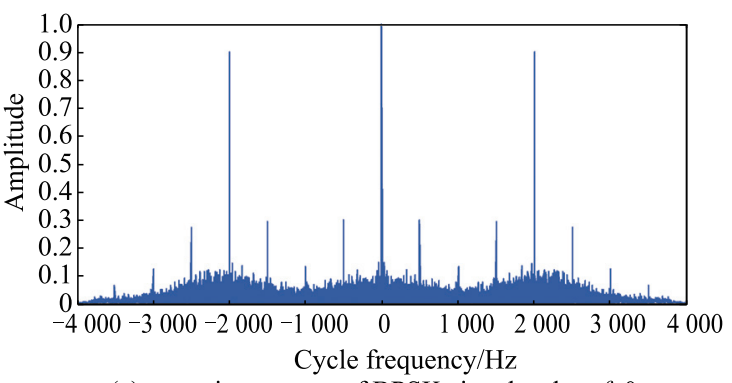

(a) $\alpha$-section spectra of BPSK signals when $f=0$ 


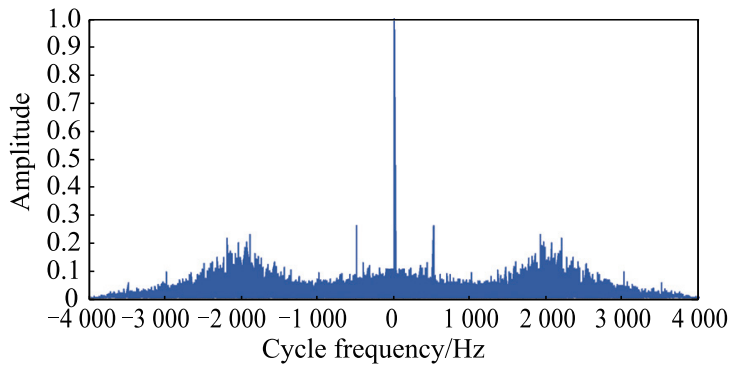

(b) $\alpha$-section spectra of QPSK signals when $f=0$

Fig. $3 \alpha$-section of BPSK/QPSK signal cycle spectra when $f=0$

$\alpha$-section of the cyclic spectrum when $f=f_{c}$ is

$$
\left|S_{x}^{\alpha}\left(f=f_{c}\right)\right|=\left\{\begin{array}{l}
\frac{|Q(\alpha / 2)|^{2}}{4 T}, \quad \alpha=0, \pm \frac{1}{T} . \\
0, \quad \alpha=\text { else }
\end{array}\right.
$$

The first item is close to a small value of 0 . In the second item, the cyclic spectrum has a peak value when $\alpha= \pm 1 / T$, and the second largest value when $\alpha= \pm 1 / T$. Thus, we search the maximum value when $f=f_{c}$. At this point the search to a maximum value corresponds to the symbol rate.

We can make use of these properties to calculate the carrier frequency and the symbol rate. The following conclusions can be drawn.

(i) The cyclic spectrum is a two-dimensional transformation, and the two parameters are spectrum frequency $\alpha$ and frequency $f[30]$.

(ii) The $\alpha$-section of the cyclic spectrum when $f=0$ has a maximum value at $\alpha= \pm 2 f_{c}$.

(iii) The $\alpha$-section of the cyclic spectrum when $f=f_{c}$ has a maximum value at $\alpha= \pm 1 / T$.

\subsection{Improved discrete spectral lines algorithm}

The above is an ideal infinite loop spectral characteristics in the case of the MPSK signal sequence, when the limited observational data length, interference term signal and noise will affect the spectral characteristics of the MPSK signal cycle spectrum.

In order to reduce the influence of truncated noises, an estimation method based on the discrete spectral line is given by the cyclic spectrum [31]. The cyclic spectrum of signals is a nonlinear transformation. The cyclic spectrum not only reflects the spectrum features of symbol rate information, but also truncates the noises, affecting the extraction of spectrum features. This effect is evident especially when the data length is limited. The extraction method of discrete spectral lines is promoted in the paper, which can suppress truncation noises, improve estimation accuracy and reduce estimation variance.
The significant difference between discrete spectral lines and lines on both sides of adjacent amplitudes is shown in Fig. 4, indicating that the background noise amplitude difference between adjacent locations is small. Therefore, it is applicable to make full use of the salient features of the projection of discrete frequency components in their neighborhoods, which is different from continuous noise, to estimate the carrier frequency and the symbol rate. The influence of truncation noises needs to be suppressed in order to improve the anti-jamming ability of noises and improve the accuracy. An improved move average compare (MAC) discrete spectrum line extraction algorithm is proposed in the paper, which is able to improve the detecting ability of discrete spectrum lines and overcome the influence caused by noises. In order to reflect the corresponding improvement of the amplitude value of discrete lines in the algorithm, we replace the original amplitude with the ratio of the square of the amplitude $a(i)$ and take length $L$ before and after that point of the neighborhood. Then the weighted average of the amplitude is expressed by the following formula:

$$
a(i)=\frac{|a(i)|^{2}}{\left(\sum_{n=i-L}^{i+L} c(n)|a(n)|\right)^{2}}
$$

where $c(n)=1 /(n-i+L+1)$. Since the uncorrelated noise is suppressed after the improved discrete spectral lines algorithm, the relevant frequency and symbol rate information will be highlighted.

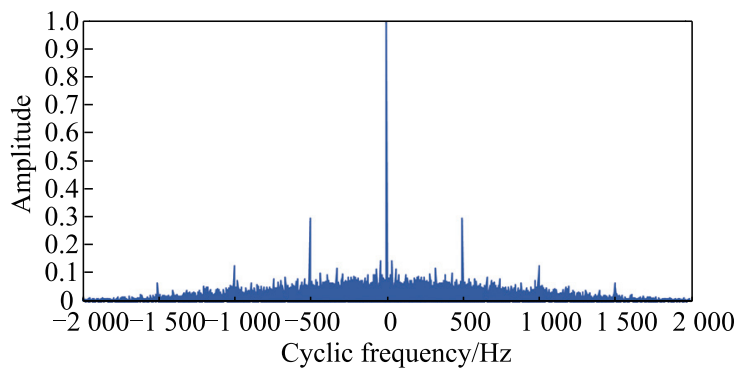

(a) $\alpha$-section spectra of BPSK signals when $f=f_{c}$

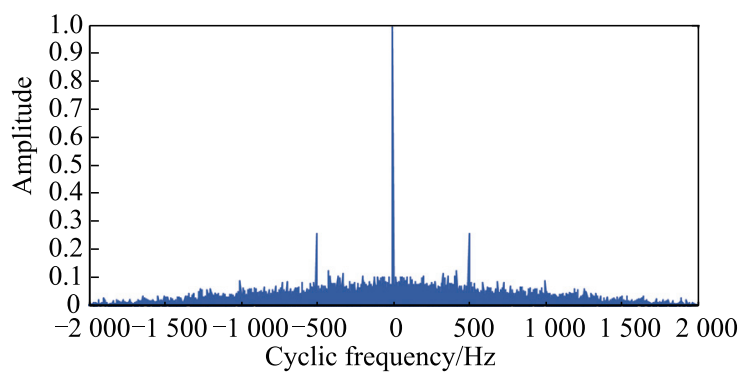

(b) $\alpha$-section spectra of QPSK signals when $f=f_{c}$

Fig. $4 \alpha$-section of BPSK/QPSK signal cyclic spectra when $f=f_{c}$ 
The simulation diagram of the BPSK signal below is under the condition that the signal to noise ratio (SNR) is $5 \mathrm{~dB}$, Fig. 5(b) is obtained by direct MAC with an improved operation. Fig. 5 clearly shows that the frequency components of Fig. 5(b) is more obvious (the symbol rate information), making it easier to search the peak. For Fig. 5(a), some of the components caused by noises will be treated as a peak, resulting error estimation of the symbol rate, reflecting the advantages of the above data processing.

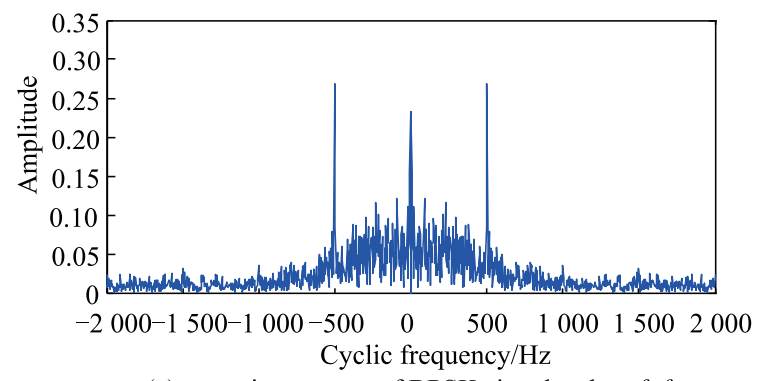

(a) $\alpha$-section spectra of BPSK signals when $f=f_{c}$

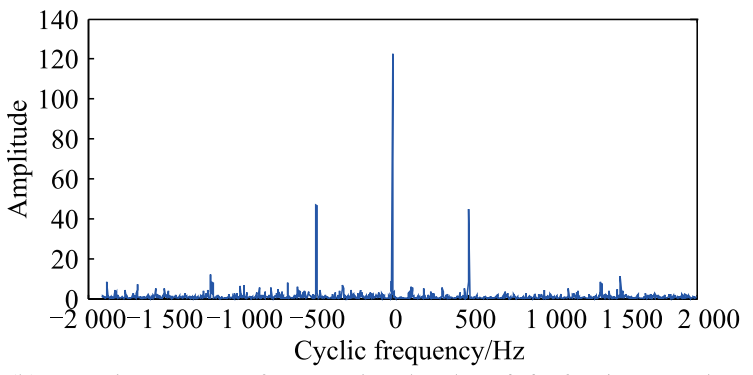

(b) $\alpha$-section spectra of BPSK signals when $f=f_{c}$ after improved MAC

Fig. $5 \alpha$-section of BPSK signal cycle spectra when $f=f_{c}$

\subsection{Parameters estimation value obtaining}

Step 1 The cyclic spectrum of the received signal is calculated by the observation data.

Step 2 According to the cyclic spectrum, the $\alpha$ cross section of $S_{x}^{\alpha}(0)$ is intercepted, the cross section is optimized, the maximum value is searched and the position of the frequency is recorded as $L_{c \max 1}$ and $L_{c \max 2}$. The carrier frequency estimation formula is $\widehat{f}_{c}=\left(\left|L_{c \max 1}\right|+\right.$ $\left.\left|L_{c \max 2}\right|\right) / 4$.

Step 3 According to the theoretical analysis, the nonstationary signal has a value only when $\alpha=0$, and the rest results are zero. Therefore, we intercept the $\alpha$ cross section of $S_{x}^{\alpha}\left(f=f_{c}\right)$ when $f=f_{c}$, then optimize the intercept section of $\alpha$. Search the maximum value of the cross section $L_{d \max 1}$ and $L_{d \max 2}$ except the circulating frequency which is $0 \mathrm{~Hz}$, and the symbol rate estimation formula is $\widehat{f_{d}}=\left(\left|L_{d \max 1}\right|+\left|L_{d \max 2}\right|\right) / 2$.

\section{Simulation results and analysis}

Carrier frequency and sign rate estimation are influenced by SNR, modulation mode and data length, which are analyzed through experiments in this section. The simulation experiment is carried out to illustrate the effectiveness of the algorithm and verify the performance of the algorithm under the AWGN channel model. The normalized mean square error (NMSE) of the parameter estimation is the evaluation criterion in this paper. The formula for the normalized MSE is given below, where the true value for the carrier frequency is $f_{c}$ and the symbol rate is $f_{d}$. $f_{c i}$ and $f_{d i}$ represent the value estimation of the carrier frequency and the symbol rate.

$$
\begin{aligned}
& \operatorname{NMSE}\left(f_{c}\right)=\frac{1}{N} \sum_{i=1}^{N}\left(\frac{f_{c i}-f_{c}}{f_{c}}\right)^{2} \\
& \operatorname{NMSE}\left(f_{d}\right)=\frac{1}{N} \sum_{i=1}^{N}\left(\frac{f_{d i}-f_{d}}{f_{d}}\right)^{2}
\end{aligned}
$$

Simulation parameters: sampling rate $f_{s}=24000 \mathrm{~Hz}$, carrier frequency $f_{c}=3000 \mathrm{~Hz}$, symbol rate $f_{b}=$ 2400 baud. The smoothing window length is 64 estimated by the frequency domain smoothing method. Five hundred independent Monte Carlo experiments are performed at different SNRs.

Experiment 1 Simulate the normalized MSE of carrier frequency and symbol rate estimation for different modulation modes such as signal 2ASK, 2FSK, BPSK and QPSK under different SNRs. The data length takes $N=5120$ sample points.

According to the previous theory, the discrete lines of the 2ASK signal and the 2FSK signal are the most obvious, so that carrier frequency estimation normalized MSE has been reduced to zero as the SNR is $-4 \mathrm{~dB}$. The carrier frequency estimation error of BPSK and QPSK signal can be reduced to zero in the SNR of $-2 \mathrm{~dB}$ in Fig. 6, and the NMSE of the symbol rate estimation of the 2ASK signal is not higher than $10^{-2}$ at an SNR of $6 \mathrm{~dB}$.

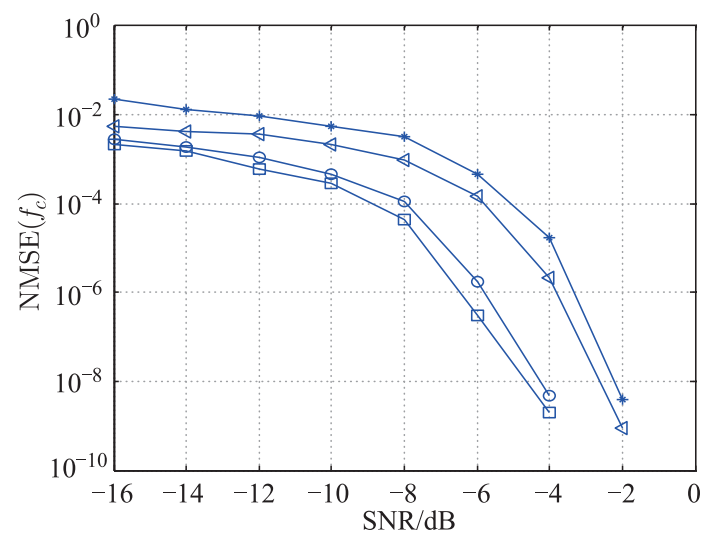

(a) NMSE of carrier frequency estimation for all different modulation signals 


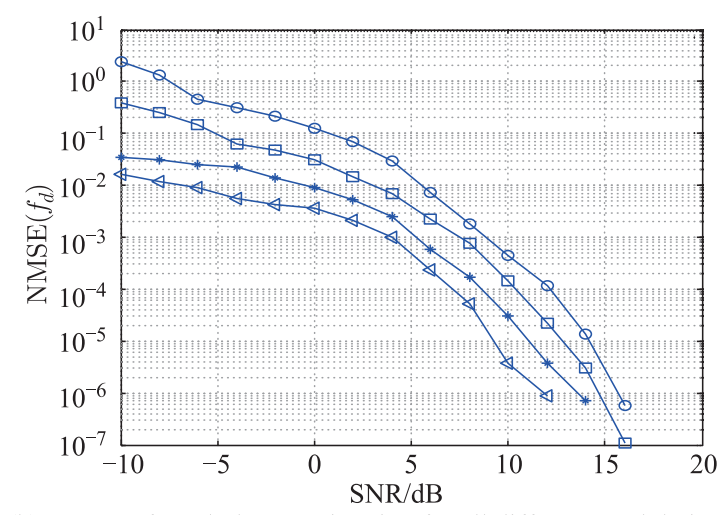

(b) NMSE of symbol rate estimation for all different modulation signals

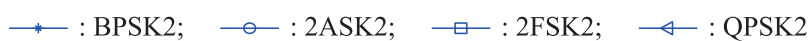

Fig. 6 NMSE of carrier frequency and symbolic rate estimation for different modulation signals

The 2 FSK signal is $4 \mathrm{~dB}$. The NMSE of the BPSK signal is not higher than $10^{-3}$ when the SNR is $6 \mathrm{~dB}$, and the QPSK signal is $4 \mathrm{~dB}$.

Experiment 2 Compare the influence of different data lengths on the error of carrier frequency and symbol rate estimation. Data lengths are taken as $N=5120,8000$, 12000 for simulation.

It is shown in Fig. 7 that the increase of data length can greatly improve the algorithm performance and reduce the MSE in parameter estimation. When $N=5120$, the carrier frequency is accurately estimated when $\mathrm{SNR}>-2 \mathrm{~dB}$ and the symbol rate is accurately estimated when $\mathrm{SNR}>$ $6 \mathrm{~dB}$; when $N=8000$, the carrier frequency is accurately estimated when $\mathrm{SNR}>-4 \mathrm{~dB}$ and the symbol rate is accurately estimated when $\mathrm{SNR}>4 \mathrm{~dB}$; when $N=12000$, the carrier frequency is accurately estimated when $\mathrm{SNR}>$ $-6 \mathrm{~dB}$ and symbol rate is accurately estimated when $\mathrm{SNR}>2 \mathrm{~dB}$. The longer the data length is, the better the performance of the algorithm will be.

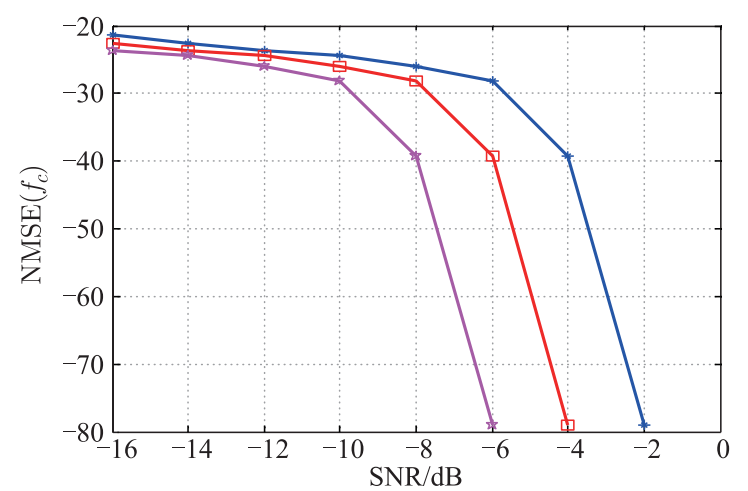

(a) NMSE of carrier frequency estimation for different signal lengths of the modulation signal

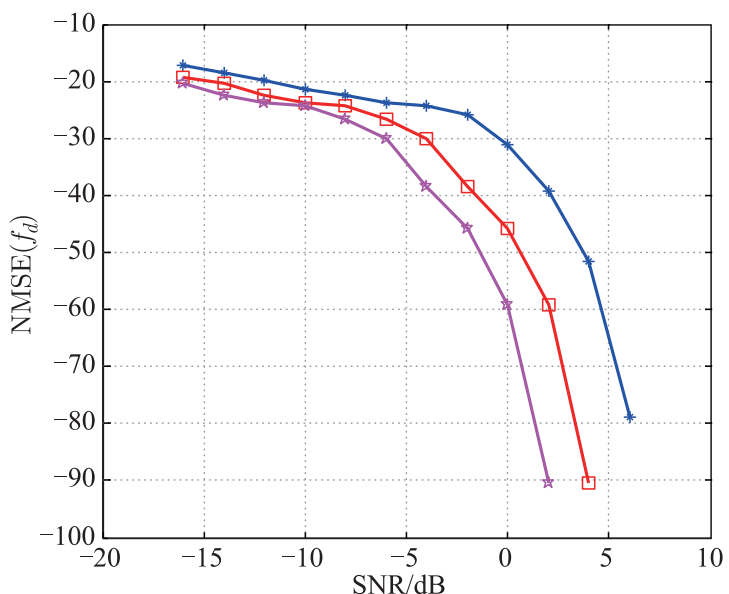

(b) NMSE of symbol rate estimation for different signal lengths of the modulation signal

$$
\longrightarrow \text { : BPSK, } N=5120 \text {; }
$$

$\longrightarrow$ : BPSK, $N=12000$.

$$
\longrightarrow \text { : BPSK, } N=8000 \text {; }
$$

Fig. 7 NMSE of parameter estimation for different signal lengths of the modulated signal

The reason is that cyclic cumulants and cyclic spectrum essentially reflect the statistic features of signals, of which the anti-noise performance advances gradually. In practical treatment, calculation is made with limited length, in which the theoretical value is closer, the induced truncation noise is smaller, the accuracy of parameter estimation is higher and the MSE is smaller while the data length is longer.

Experiment 3 Compare the performance of carrier frequency and symbol rate estimation by the cyclic spectrum algorithm and the improved algorithm in this paper.

The section of cyclic spectra is used to estimate the carrier frequency and symbol rate. As shown in Fig. 8, BPSK1 represents the original algorithm, and BPSK2 represents the improved algorithm. The improved algorithm improves the estimation accuracy and reduces the NMSE. As the SNR grows, the estimation result gets better. Accurate estimation of symbol rate estimation is at $\mathrm{SNR}>6 \mathrm{~dB}$ and carrier frequency estimation is at $\mathrm{SNR}>-2 \mathrm{~dB}$. Thus, the algorithm has a better anti-noise performance. This is because the additive Gaussian noise is stationary. The proposed algorithm is used to estimate the discrete spectrum of the carrier frequency and bit rate. The additive Gaussian noise at the spectral cross section is zero, thus effectively overcoming the effect of noises. For the background noise introduced by the data truncation, the improved MAC algorithm is used to enhance the discrete spectrum at the cyclic frequency, which overcomes the influence of the background noise. Thus, the overall anti-noise performance of the algorithm is the best. 


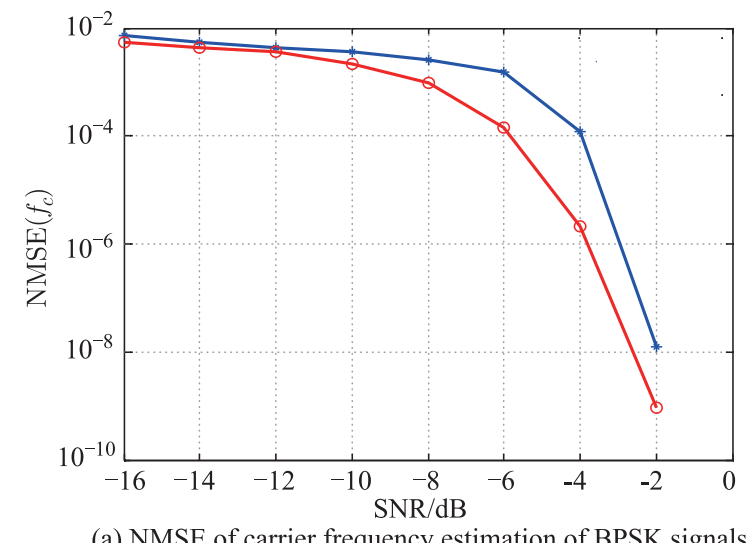

(a) NMSE of carrier frequency estimation of BPSK signals

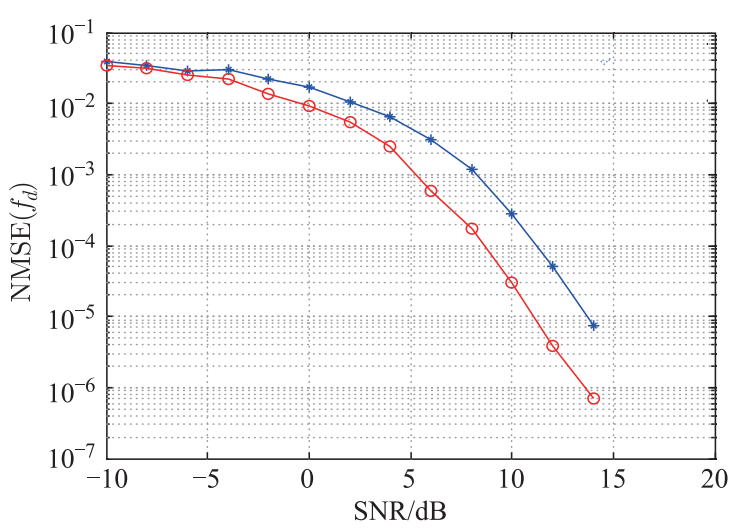

(b) NMSE of symbol rate estimation of BPSK signals

$$
\longrightarrow \text { : BPSK1; } \multimap \text { : BPSK2. }
$$

Fig. 8 NMSE of carrier frequency and symbol rate estimation of BPSK signals

\section{Conclusions}

In this paper, the problem of parameter estimation in modulation recognition is studied. The estimation of the parameters mainly includes the carrier frequency estimation and the symbol rate estimation. Considering that the cyclic spectrum method has a good anti-noise performance and can be locally calculated, the carrier frequency and symbol rate can be estimated by different cross sections of the cyclic spectrum. In the process of line extraction, an improved discrete spectral line extraction algorithm is introduced. The simulation results show that the improved method has a better performance than the original method and improves the estimation performance.

\section{References}

[1] DOBRE O A, ABDI A, BAR-NESS Y, et al. Survey of automatic modulation classification techniques: classical approaches and new trends. IET Communications, 2007, 1(2): $137-156$.

[2] SPOONER C M, GARDNER W A. Robust feature detection for signal interception. IEEE Trans. on Communications, 1994, 42(5): $2165-2173$.
[3] HAYKIN S. Cognitive radio: brain-empowered wireless communications. IEEE Journal on Selected Areas in Communications, 2005, 23(2): $201-220$.

[4] YUCEK T, ARSLAN H. A survey of spectrum sensing algorithms for cognitive radio applications. IEEE Communications Surveys \& Tutorials, 2009, 11(1): 116-130.

[5] ČBRIĆ D B. Cognitive radios: system design perspective. Berkeley: University of California Berkeley, 2007.

[6] CABRIC D. Addressing feasibility of cognitive radios. Signal Processing Magazine IEEE, 2008, 25(6): 85-93.

[7] ZHANG Q, DOBRE O A, ELDEMERDASH Y A, et al. Second-order cyclostationarity of BT-SCLD signals: theoretical developments and applications to signal classification and blind parameter estimation. IEEE Trans. on Wireless Communications, 2013, 12(4): $1501-1511$.

[8] LIM C W, WAKIN M B. Automatic modulation recognition for spectrum sensing using nonuniform compressive samples. Proc. of the 2012 IEEE International Conference on Communications, 2012: $3505-3510$.

[9] WU Z, YANG T C. Blind cyclostationary carrier frequency and symbol rate estimation for underwater acoustic communication. Proc. of the IEEE International Conference on Communications, 2012: 3482 - 3486.

[10] COTAE P, YANG T C. A cyclostationary blind Doppler estimation method for underwater acoustic communications using direct-sequence spread spectrum signals. Proc. of the 8th IEEE International Conference on Communications, 2010: $323-$ 326.

[11] LIKE E, CHAKRAVARTHY V, RATAZZI, P, et al. Signal classification in fading channels using cyclic spectral analysis. Eurasip Journal on Wireless Communications and Networking, 2009, 2009(1): 1-14.

[12] MAJHI S, HO T S. Blind symbol-rate estimation and test bed implementation of linearly modulated signals. IEEE Trans. on Vehicular Technology, 2015, 64(3): 954-963.

[13] SANDERSON J, LI X, LIU Z, et al. Hierarchical blind modulation classification for underwater acoustic communication signal via cyclostationary and maximal likelihood analysis. Proc. of the Military Communications Conference, 2014: 2934.

[14] ZHOU R, LI X, YANG T C, et al. Real-time cyclostationary analysis for cognitive radio via software defined radio. Proc. of the Global Communications Conference, 2012: 1495-1500.

[15] QU Y, LI X, ZHOU R, et al. Software-defined radio based automatic blind hierarchical modulation detector via secondorder cyclostationary analysis and fourth-order cumulant. Proc. of the Military Communications Conference, 2013: $441-446$.

[16] GAPPMAIR W, SCHLEMMER H, GINESI A. Joint synchronization of symbol timing and carrier frequency using the extended zero-crossing property. Proc. of the Advanced Satellite Multimedia Systems Conference and the Signal Processing for Space Communications Workshop, 2016: 1-8.

[17] ZHU X, ZHANG G. A modified carrier frequency estimation method based on self-correlation for QPSK signal. Proc. of the International Conference on Automatic Control and Artificial Intelligence, 2012: 129-132.

[18] ZAPLATA F, KASAL M. Spectrum averaging carrier frequency estimation. Proc. of the 25th International Conference Radioelektronika, 2015: 264-267.

[19] GONG M D, GUO R H. Carrier frequency estimation based on second-order cyclic cumulants. Computer Engineering, 2011, 37(20): $81-82,86$.

[20] SUN X, FENG Q. A method of carrier frequency estimation based on spectral analysis of digital communication signal. 
Proc. of the IEEE International Conference on Computer Science and Information Technology, 2010: 67-70.

[21] ZHANG Z, LI L, XIAO X. Carrier frequency and chip rate estimation based on cyclic spectral density of MPSK signals. Proc. of the International Conference on Communications, Circuits and Systems, 2004: 859-862.

[22] LIU K, ZUO Z Y, HUANG Q. A symbol rate estimation algorithm for linearly mixed digital modulation signals. Proc. of the IET International Communication Conference on Wireless Mobile and Computing, 2010: 233-236.

[23] TIAN Z, TAFESSE Y, SADLER B M. Cyclic feature detection with sub-nyquist sampling for wideband spectrum sensing. IEEE Journal of Selected Topics in Signal Processing, 2012, 6(1): 58-69.

[24] COHEN D, POLLAK L, ELDAR Y C. Carrier frequency and bandwidth estimation of cyclostationary multiband signals. Proc. of the IEEE International Conference on Acoustics, Speech and Signal Processing, 2016: 3716-3720.

[25] LIU S P, WEN X, JIN L. A new nonlinear filtering algorithm for colored background self-noises suppressing of symbol rate estimation. Chinese Journal of Electronics, 2007, 35(1): 95 99. (in Chinese)

[26] DOBRE O A, ABDI A, BAR-NESS Y, et al. Cyclostationaritybased modulation classification of linear digital modulations in flat fading channels. Wireless Personal Communications, 2010, 54(4): 699-717.

[27] VUKOTIC S, VUCIC D. Detection and clasiffication of OFDM/QAM and OFDM/OQAM signals based on cyclostationary features. Proc. of the 23rd Telecommunications Forum Telfor, 2015: $232-235$.

[28] TUYEN LE A, TRAN L C, HUANG X. Cyclostationary analysis of analog least mean square loop for self-interference cancellation in in-band full-duplex systems. IEEE Communications Letters, 2017, 21(12): $2738-2741$.
[29] XU W, WANG B. Parameter estimation of time-frequency overlapped signals based on cyclic spectrum. Journal of Data Acquisition \& Processing, 2014, 29(3): 456-464.

[30] YU G C, TAO L, ZHEN H, et al. An improved cyclic spectrum detection algorithm in CR systems. Journal of Beijing University of Posts \& Telecommunications, 2009, 32(2): 89-92.

[31] MAZET L, LOUBATON P. Cyclic correlation based symbol rate estimation. Record of the 33rd Asilomar Conference on Signals, Systems and Computers, 1999, 2: $1008-1012$.

\section{Biographies}

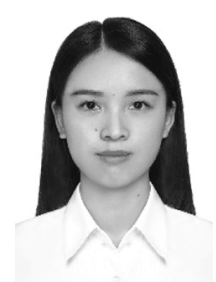

CAO Sisi was born in in 1991. She received her bachelor's degree in communication engineering in 2014 and master's degree in the field of electronics and communication engineering in 2017 both from University of Chongqing, Chongqing, China. Her research insterest is non-cooperative communication signal processing. Currently she is working as an assistant engineer employed by the Institute of Computer Applications, China Academy of Engineering Physics.

E-mail: 609569884@qq.com

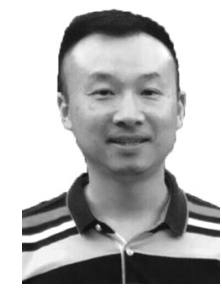

ZHANG Weiyan was born in 1978. He is a senior engineer in Institute of Computer Applications, China Academy of Engineering Physics. His research interest is database technology information security. E-mail: zwy5552@dingtalk.com 